\title{
Desempenho do parasitóide Nasonia vitripennis (Walker) (Hymenoptera, Pteromalidae) utilizando como hospedeiro Cochliomyia macellaria (Fabricius) (Diptera, Calliphoridae), sob diferentes tempos de exposição
}

\author{
Leandro Silva Barbosa ${ }^{1,2}$, Márcia Souto Couri ${ }^{2,4} \&$ Valéria Magalhães Aguiar Coelho ${ }^{3}$
}

${ }^{1}$ Programa de Pós-graduação em Ciências Biológicas (Zoologia), Museu Nacional, Universidade Federal do Rio de Janeiro, Quinta da Boa Vista, 20940-040 Rio de Janeiro-RJ, Brasil. leanbarbosa@globo.com

${ }^{2}$ Laboratório de Diptera, Departamento de Entomologia, Museu Nacional, Universidade Federal do Rio de Janeiro, Quinta da Boa Vista, 20940-040 Rio de Janeiro-RJ, Brasil.mcouri@terra.com.br

${ }^{3}$ Laboratório de Estudo de Dípteros, Departamento de Microbiologia e Parasitologia, Universidade Federal do Estado do Rio de Janeiro, Rua Frei Caneca 94 , 20211-040 Rio de Janeiro-RJ, Brasil. valeria@unirio.br

${ }^{4}$ Bolsista de Produtividade em Pesquisa 1A, CNPq

\begin{abstract}
Performance of the parasitoid Nasonia vitripennis (Walker) (Hymenoptera, Pteromalidae) using as host Cochliomyia macellaria (Fabricius) (Diptera, Calliphoridae) under different exposition times. The post-embryonic development, the productivity of the host pupae, the rate of parasitism and the sex ratio of Nasonia vitripennis reared in pupae of Cochliomyia macellaria were studied. Different exposition times were used: 24, 48, 72, 96 hours. Nulliparous females originating from the stock colony were individualized in test tubes covered with hydrophobic cotton and containing the host pupae. Each treatment was constituted by 10 replicates. After exposition, the hymenopterans were discarded and the host pupae were individualized in test tubes until the emergency of the adults of C. macellaria or N. vitripennis. Samples of host pupae not exposed to parasitism and receiving the same experimental treatment, were used as control. The parasitoids showed a slower development in relation to the increase exposition time. The 72 hours exposition was the one which enabled higher productivity. The sex ratio tended towards a deviation for the birth of females after 48 hour. The rate of parasitism increased up to 72 hours. The rate of dipteran's emergence showed a decrease until 72 hours.
\end{abstract}

KEYWORDS. Biological Control; fly; parasitism.

RESUMO. Desempenho do parasitóide Nasonia vitripennis (Walker) (Hymenoptera, Pteromalidae) utilizando como hospedeiro Cochliomyia macellaria (Fabricius) (Diptera, Calliphoridae), sob diferentes tempos de exposição. Foram estudados o desenvolvimento pós-embrionário, a produtividade da pupa hospedeira, a taxa de parasitismo e a razão sexual de Nasonia vitripennis (Walker) criadas em pupas hospedeiras de Cochliomyia macellaria (Fabricius). Analisaram-se diferentes tempos de exposição: 24 , 48, 72 e 96 horas. Fêmeas nulíparas foram individualizadas em tubos de ensaio contendo as pupas hospedeiras e tampados com algodão hidrófobo. Cada tratamento foi constituído de 10 repetições. Após a exposição, os himenópteros foram descartados e as pupas hospedeiras foram mantidas individualizadas. $\mathrm{O}$ controle consistiu de pupas de C. macellaria não expostas ao parasitismo utilizando-se o mesmo delineamento experimental. Os parasitóides apresentaram um desenvolvimento pós-embrionário mais lento com aumento do tempo de exposição. A exposição mais produtiva foi a de 72 horas. A razão sexual apresentou uma tendência ao nascimento de fêmeas após a exposição de 48 horas. A taxa de parasitismo apresentou crescimento até a exposição de 72 horas. A taxa de nascimento de dípteros também apresentou queda até o tempo de exposição de 72 horas.

PALAVRAS-CHAVE. Controle Biológico; moscas; parasitismo.

Estudos sobre a viabilidade do uso de Cochliomyia macellaria (Fabricius 1775) (Diptera, Calliphoridae) como hospedeiro do parasitóide Nasonia vitripennis (Walker, 1836) (Hymenoptera, Pteromalidae) foram publicados recentemente por Barbosa et al. (2008 a, b) visando contribuir para o conhecimento do controle biológico de dípteros muscóides, como também para um melhor entendimento sobre a dinâmica populacional deste díptero na natureza.

Após a invasão e rápida dispersão do gênero exótico Chrysomya Robineau-Desvoidy, 1830 nas Américas (Gagné 1981; Prado 1982), a espécie C. macellaria, antes muito comum nas áreas urbanas e rurais, vem se tornando relativamente rara nesses ambientes (Guimarães et al.
1979). Estudos posteriores efetuados em campo também observaram esse declínio populacional (d'Almeida \& Lopes 1983; Baumgartner \& Greenberg 1984; Baumgartner 1988 e Marinho et al., 2003). Testes laboratoriais observaram a interação entre larvas da espécie nativa com larvas das espécies de Chrysomya (Aguiar-Coelho et al. 1995; AguiarCoelho \& Milward-de-Azevedo 1995; 1996), verificando que as espécies exóticas possuíam vantagens competitivas durante a fase larval.

Até os trabalhos de Barbosa et al. (2008 a, b) não haviam registros sobre as interações entre C. macellaria e o parasitóide $N$. vitripennis, cujos resultados podem estar, aliados a competição larval com outros dípteros, contribuindo também 
para a redução populacional desta espécie na natureza.

Dando continuidade a esses estudos, o presente trabalho tem como objetivo avaliar o desenvolvimento pós-embrionário, produtividade de parasitóides por hospedeiro, razão sexual e taxa de parasitismo de $N$. vitripennis criados em pupas de $C$. macellaria em diferentes tempos de exposição.

\section{MATERIAL E MÉTODOS}

O estabelecimento e manutenção das colônias de $C$. macellaria e $N$. vitripennis foi detalhada em Barbosa et al. (2008 a).

Fêmeas nulíparas de $N$. vitripennis foram individualizadas em tubos de ensaio tampados com algodão hidrófobo, contendo cinco pupas hospedeiras, mantidos a $27{ }^{\circ} \mathrm{C}, 60 \pm$ $10 \%$ de umidade relativa, 14 horas de fotofase e supridos com solução aquosa de mel (2:1) em tira de papel filtro. Lotes com 10 tubos de ensaio foram submetidos à diferentes tempos de exposição às fêmeas, em quatro tratamentos: 24, 48, 72 e 96 horas. Como controle, 40 tubos de ensaio não foram expostos à fêmeas para se avaliar a taxa de mortalidade.Após a exposição às vespas foram descartadas e as pupas hospedeiras individualizadas em tubos de ensaio, alocados aleatoriamente em bandejas onde se aguardou a emergência dos adultos de C. macellaria ou N. vitripennis.As observações foram diárias e realizadas sempre em mesmo horário, até o $35^{\circ}$ dia após o término da exposição.

Analisaram-se dados referentes à duração do período do desenvolvimento de ovo a emergência, produtividade de parasitóides por hospedeiro, razão sexual e taxa de parasitismo. Considerou-se a produtividade por hospedeiro o número de parasitóides emergidos por pupa hospedeira, enquanto a taxa de parasitismo o número de pupas de $C$. macellaria que originaram parasitóides.

Para avaliar os resultados foram utilizados a Análise de Variância e o Teste T-studend (Magalhães \& Lima 2001).

\section{RESULTADOS}

A duração média do período de desenvolvimento de ovo a emergência de machos e fêmeas de $N$. vitripennis submetidas a diferentes tempos de exposição ao hospedeiro $(24,48,72$ e 96 horas) variou significativamente entre as repetições pela análise de variância $(\mathrm{P}<0,0001)$. Não foi observada diferença significativa entre as exposições 24,48 e 72 horas, a exposição de 96 horas apresentou significativamente a maior duração (15,85 dias) (Tabela I).

Para as fêmeas observou-se um aumento do desenvolvimento em função do aumento do tempo de exposição. Em contrapartida, nos machos o maior tempo de desenvolvimento observado foi na exposição de 24 horas (18,90 dias) diferindo significativamente das demais (Tabela I).

Observa-se na Figura 1 o ritmo de emergência de $N$. vitripennis com pico no $14^{\circ}$ dia após a exposição do parasitóide ao hospedeiro por 24, 48 e 72 horas. Na exposição de 96 horas foi observado um retardo no pico de emergência para o $16^{\circ}$ dia.
A produtividade de machos e fêmeas de $N$. vitripennis criadas em pupas de $C$. macellaria utilizando-se a relação 1 parasitóide: 5 hospedeiros submetidos a diferentes tempos de exposição (24, 48, 72 e 96 horas) variou significativamente pela ANOVA $(\mathrm{F}=3,57$ e $\mathrm{P}=0,016)$. Observou-se que a produção de parasitóides foi significativamente maior na exposição de 72 horas em relação à de 48 horas não diferindo entre as demais (Tabela II).

Não foi observada diferença significativa entre as produtividades de machos nos diferentes tempos de exposição. Porém, a maior produção de fêmeas foi observada no tempo de exposição de 72 horas em relação aos demais.

Houve desvio da razão sexual com um maior nascimento de fêmeas nos diferentes tempos de exposição do hospedeiro ao parasitóide (Tabela II). A curva de tendência exponencial mostrou um aumento do nascimento de fêmeas de $N$. vitripennis com o aumento do tempo de exposição (Fig. 2).

Observou-se maior taxa de parasitismo de C. macellaria expostas a $N$. vitripennis por 72 horas, seguido de 96, 48 e 24 horas. A taxa de pupas inviáveis apresentou aumento em função do tempo de exposição. Já a de emergência de dípteros decresceu até 72 horas, com um aumento em 96 horas. O controle apresentou a taxa de emergência de $98 \%$ (Fig. 3).

\section{DISCUSSÃO}

Os resultados relativos ao desenvolvimento de $N$. vitripennis criadas em pupas de C. macellaria, utilizando diferentes tempos de exposição do hospedeiro ao parasitóide, mostraram uma leve aceleração do desenvolvimento de ovo a emergência do adulto até a exposição de 72 horas, voltando a declinar em 96 horas. Podemos também observar essa tendência verificando o início da emergência, onde o tratamento de 72 horas foi o que começou a emergir primeiro.

Esse padrão nos machos não pôde ser observado devido à emergência de 17 machos no $28^{\circ}$ dia após o início da exposição no tratamento de 24 horas, o que contribuiu para uma maior média da extensão do desenvolvimento. A capacidade de $N$. vitripennis produzir larvas diapáusicas (Whiting 1967) deve ser considerada neste caso. Fatores ambientais tais como:

Tabela I. Duração média do período de desenvolvimento de ovo a emergência (em dias) de Nasonia vitripennis criadas em pupas de Cochliomyia macellaria expostas por diferentes períodos ao parasitismo, na proporção 1 parasitóide ${ }^{1}$ : 5 hospedeiros $^{2}\left(27^{\circ} \mathrm{C}, 60 \%\right.$ U.R. e 14 horas de fotofase $)$.

\begin{tabular}{cccccccccc}
\hline \multirow{2}{*}{$\begin{array}{c}\text { Tempo } \\
\text { de } \\
\text { Expo- } \\
\text { sição }\end{array}$} & \multicolumn{6}{c}{ Machos } & \multicolumn{7}{c}{ Duração do desenvolvimento (dias) } \\
\cline { 2 - 11 } & $\pm \mathrm{dp}$ & $\mathrm{IV}$ & $\mathrm{X}$ & $\pm \mathrm{dp}$ & $\mathrm{IV}$ & $\mathrm{X}$ & $\pm \mathrm{dp}$ & $\mathrm{IV}$ \\
\hline $24 \mathrm{~h}$ & $18,90_{\mathrm{a}}$ & 6,84 & $13-28$ & $13,95_{\mathrm{a}}$ & 0,69 & $13-16$ & $14,94_{\mathrm{a}}$ & 3,67 & $13-28$ \\
$48 \mathrm{~h}$ & $14,91_{\mathrm{b}}$ & 1,10 & $13-18$ & $14,64_{\mathrm{b}}$ & 1,08 & $13-20$ & $14,77_{\mathrm{a}}$ & 1,08 & $13-20$ \\
$72 \mathrm{~h}$ & $14,95_{\mathrm{b}}$ & 0,14 & $12-19$ & $14,61_{\mathrm{b}}$ & 1,33 & $12-19$ & $14,66_{\mathrm{a}}$ & 1,35 & $12-19$ \\
$96 \mathrm{~h}$ & $15,84_{\mathrm{b}}$ & 1,15 & $14-20$ & $15,85_{\mathrm{c}}$ & 1,22 & $14-21$ & $15,85_{\mathrm{b}}$ & 1,22 & $14-21$ \\
\hline
\end{tabular}

$\mathrm{X}=$ média, $\mathrm{dp}=$ desvio padrão, $\mathrm{IV}=$ intervalo de variação. Médias seguidas pela mesma letra não diferem significativamente pelo teste T em nível de $5 \%$ de significância. ${ }^{1}$ Fêmeas nulíparas individualizadas $(n=10 /$ tratamento) com até 24 horas pós-emergência. ${ }^{2}$ Pupas com até 24 horas de idade. 


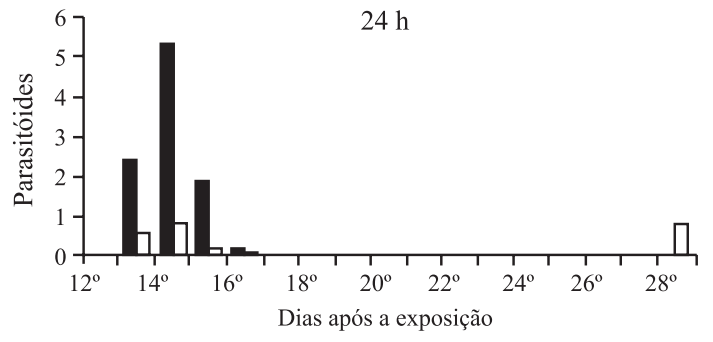

$72 \mathrm{~h}$

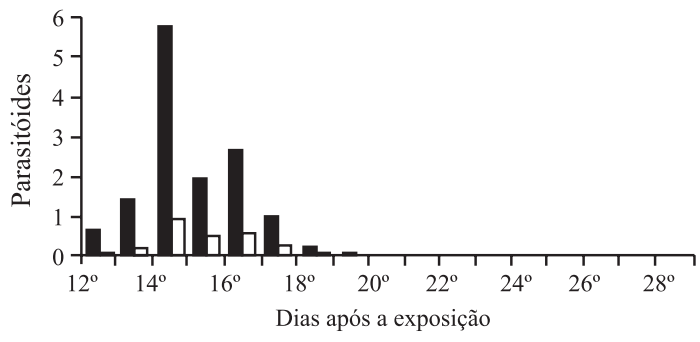

Fêmeas

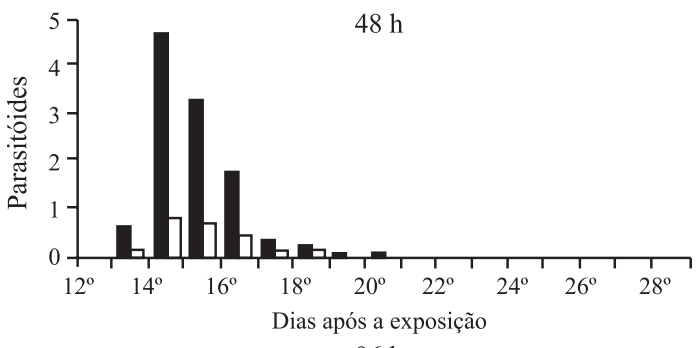

$96 \mathrm{~h}$

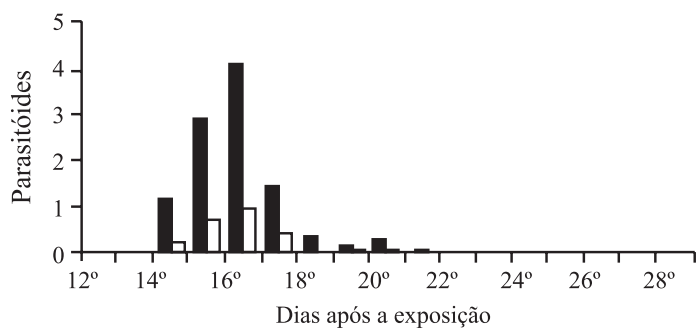

Machos

Fig. 1. Ritmo de emergência de adultos de Nasonia vitripennis criadas em pupas de Cochliomyia macellaria expostas por 72 horas a fêmeas nulíparas por diferentes períodos ao parasitismo, na proporção 1 parasitóide: 5 hospedeiros $\left(27^{\circ} \mathrm{C}, 60 \%\right.$ U.R. e 14 horas de fotofase)

temperatura, fotoperíodo, umidade, espécie hospedeira e idade da fêmea parasitóide podem induzir este tipo de comportamento estratégico (Wylie 1963; Walker \& Pimentel 1966 e Saunders et al. 1970).

Cardoso \& Milward-de-Azevedo (1995) estudando a exposição de C. megacephala à $N$. vitripennis por períodos de 24 e 48 horas, não verificaram diferença significativa no tempo de desenvolvimento entre os dois tempos de exposição.

Os picos de emergência de parasitóides, no presente estudo, tenderam a ser posteriores com o aumento do tempo de exposição, estes se apresentaram compreendidos entre o $14^{\circ}$ e $16^{\circ}$ dia após a exposição do hospedeiro ao parasitóide. Gulias-Gomes et al. (2003), trabalhando com C. megacephala, a $27^{\circ} \mathrm{C}$, UR $65 \pm 10 \%$, observaram picos de emergências concentrados entre o $14^{\circ}$ e $15^{\circ}$ dias após o início da exposição. Os picos de emergência para machos e para

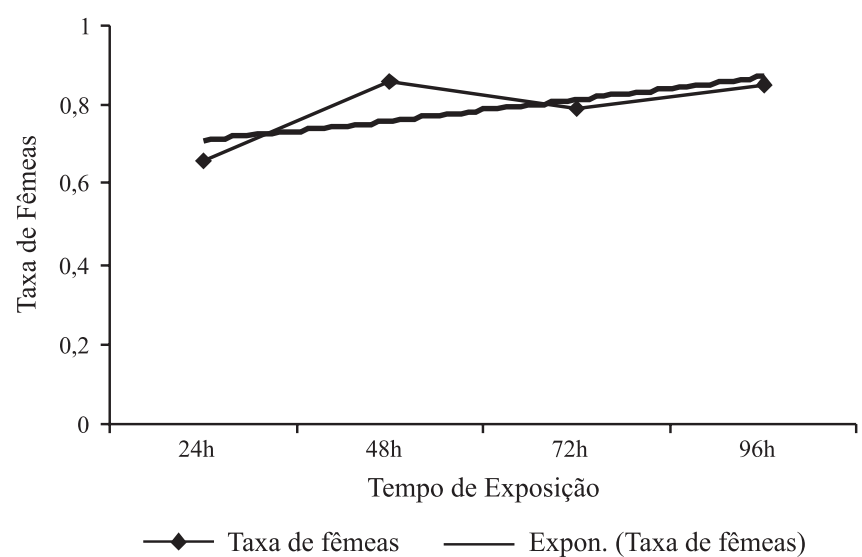

Fig. 2. Gráfico de tendência exponencial da taxa de fêmeas de Nasonia vitripennis criadas em pupas de Cochliomyia macellaria expostas por 72 horas a fêmeas nulíparas, utilizando-se diferentes tempos de exposição $\left(27^{\circ} \mathrm{C}, 60 \%\right.$ U.R. e 14 horas de fotofase). fêmeas, no presente trabalho, obedeceram à mesma tendência entre si.

Observou-se um aumento da inviabilidade de pupas hospedeiras com o aumento do tempo de exposição a partir de 48 horas alcançando o pico em 96 horas. O mesmo foi observado por Cardoso \& Milward-de-Azevedo (1995), que constataram aumento da mortalidade de pupas em densidades elevadas do hospedeiro expostas ao parasitismo por 48 horas, em comparação com 24 horas. Os autores consideraram como justificava para esta observação o efeito deletério exploratório e alimentar das fêmeas de $N$. vitripennis, provocado pela ruptura do pupário do hospedeiro. Esta ruptura através do ovipositor pode ser realizada com a finalidade de permitir a ingestão de fluído do corpo do hospedeiro ou para permitir o reconhecimento das pupas já parasitadas, apresentando-se como uma estratégia da espécie para evitar o superparasitismo. Segundo Wylie (1966), o tempo de exposição pode influenciar o estado nutricional das fêmeas parasitóides por possibilitar

Tabela II. Razão sexual e número de parasitóides adultos de Nasonia vitripennis criados em pupas de Cochliomyia macellaria expostas por diferentes períodos ao parasitismo, na proporção 1 parasitóide ${ }^{1}: 5$ hospedeiros $^{2}$ $\left(27^{\circ} \mathrm{C}, 60 \%\right.$ U.R. e 14 horas de fotofase).

\begin{tabular}{|c|c|c|c|c|c|c|c|}
\hline \multirow{3}{*}{$\begin{array}{l}\text { Tempo } \\
\text { de } \\
\text { Expo- } \\
\text { sição }\end{array}$} & \multirow{3}{*}{$\begin{array}{l}\text { Razão } \\
\text { sexual }\end{array}$} & \multicolumn{6}{|c|}{$\mathrm{N}^{\circ}$. de Parasitóides adultos } \\
\hline & & \multicolumn{2}{|c|}{ Machos } & \multicolumn{2}{|c|}{ Fêmeas } & \multicolumn{2}{|c|}{ Machos e Fêmeas } \\
\hline & & $\mathrm{X}$ & IV & $\mathrm{X}$ & IV & $\mathrm{X}$ & IV \\
\hline $24 \mathrm{~h}$ & 0,66 & $2,82_{\mathrm{a}}$ & $1-17$ & 9,65 & $1-18$ & $12,05_{\mathrm{ab}}$ & $3-20$ \\
\hline $48 \mathrm{~h}$ & 0,86 & $2,19^{a}$ & $1-4$ & $11,00_{\mathrm{ab}}^{\mathrm{a}}$ & $2-21$ & $12,23_{\mathrm{b}}^{\mathrm{ab}}$ & $2-23$ \\
\hline $72 \mathrm{~h}$ & 0,79 & $2,74_{\mathrm{a}}^{\mathrm{a}}$ & $1-7$ & $13,38_{b c}^{a b}$ & $3-24$ & $15,86^{b}$ & $4-31$ \\
\hline $96 \mathrm{~h}$ & 0,85 & $2,82_{a}^{a}$ & $1-8$ & $10,35_{\mathrm{a}}$ & $1-18$ & $12,67_{\mathrm{ab}}^{\mathrm{a}}$ & $1-23$ \\
\hline
\end{tabular}

Razão sexual=fêmea /macho+fêmea, $X=$ média, $I V=$ intervalo de variação, Médias seguidas pela mesma letra não diferem significativamente pelo teste T em nível de $5 \%$ de significância. ${ }^{1}$ Fêmeas nulíparas individualizadas ( $\mathrm{n}=$ 10 /tratamento) com até 24 horas pós-emergência. ${ }^{2}$ Pupas com até 24 horas de idade. 


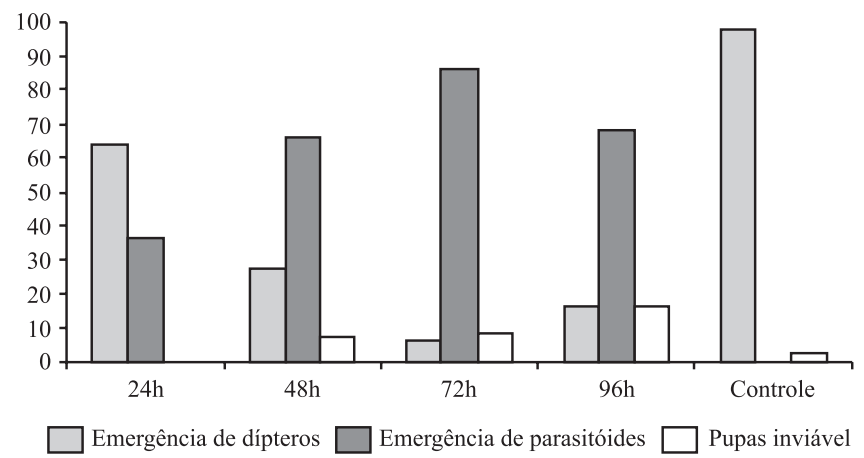

Fig. 3. Percentual de pupas com emergência de Nasonia vitripennis, pupas com emergência de adultos de Cochliomyia macellaria e pupas que não originaram adultos parasitóides /ou hospedeiros (pupas inviáveis) expostas por diferentes períodos ao parasitismo, na proporção 1 parasitóide': 5 hospedeiros ${ }^{2}\left(27^{\circ} \mathrm{C}, 60 \%\right.$ U.R. e 14 horas de fotofase). ${ }^{1}$ Fêmeas nulíparas individualizadas ( $\mathrm{n}=10$ /tratamento) com até 24 horas pós-emergência. ${ }^{2}$ Pupas com até 24 horas de idade.

maior ingestão de fluidos dos pupários hospedeiros.

Cardoso \& Milward-de-Azevedo (1995) observaram um aumento no número de parasitóides adultos produzidos por pupa hospedeira com o aumento do tempo de exposição de 24 para 48 horas. No presente trabalho, esse aumento foi observado até 72 horas, e apresentando queda em 96 horas. Estes resultados podem estar relacionados ao superparasitismo e à capacidade exploratória e alimentar da fêmea parasitóide (Cardoso \& Milward-de-Azevedo 1995; Wylie 1963). O desenvolvimento do parasitóide está intimamente associado ao tamanho (Cardoso \& Milward-de-Azevedo 1996), bem como à densidade de parasitóides e tempo de exposição do hospedeiro, já que estes fatores influenciam no tamanho da prole (Cardoso \& Milward-de-Azevedo 1995).

Muitas teorias vêm sendo apresentadas sobre a razão sexual em parasitóides. Wylie (1966) sugere que o superparasitismo é um dos fatores diretamente responsáveis pelo aumento da porcentagem de machos na prole. O gráfico de tendência exponencial observado no presente trabalho mostrou que a taxa de fêmeas na prole aumentou em função do tempo de exposição, indicando uma tendência inversa, que pode ser explicada pelo fato do superparasitismo ter sido causado pela variável tempo de exposição e não quantidade de parasitóides (Barbosa et al. 2008 a, b; Cardoso \& Milward-de-Azevedo 1995). O aumento do tempo de exposição dificulta a visualização dos efeitos do superparasitismo, devido à ação exploratória e randômica das fêmeas adultas sobre os hospedeiros (Cardoso \& Milward-de-Azevedo 1995). Outros fatores também podem influenciar na razão sexual de vespas parasitóides, como o tamanho do hospedeiro, onde os mais robustos apresentam uma tendência a um maior número de fêmeas na prole como verificado por Van den Assem et al. (1984) para Anisopteromalus calandrae (Howard) (Chalcidoidae, Pteromalidae).

Shuker et al. (2005) testaram uma hipótese chamada Asymetrical Local mate competition, que é a extensão da teoria de Hamilton (1967), Local mate competition (LMC), que sustenta a tendência ao aumento da taxa de fêmeas na prole de espécies onde apenas as fêmeas abandonam o pupário dispersando-se. Porém, a versão Shuker et al. (2005) trata de casos nos quais as fêmeas depositam seus ovos em diversos lugares, em tempos distintos, gerando emergências em tempo diferentes e encontros de machos competidores de ciclos diferentes. Neste teste, Shuker et al. (2005) verificaram que as fêmeas de $N$. vitripennis possuem uma tendência a reduzir sua taxa de fêmeas na prole caso estejam utilizando um hospedeiro já parasitado.

Os parasitóides apresentaram um desenvolvimento pósembrionário mais lento com o tempo de exposição de 96 horas. A exposição mais produtiva e que se iniciou primeiro foi a de 72 horas. A razão sexual apresentou uma tendência ao nascimento de fêmeas após a exposição de 48 horas. A taxa de parasitismo apresentou crescimento até a exposição de 72 horas. A taxa de nascimento de dípteros também apresentou queda até o tempo de exposição de 72 horas. Logo podemos concluir que, segundo os critérios de produtividade e de taxa de parasitismo, o melhor tempo de exposição foi o de 72 horas.

Agradecimentos. À Dra. Maria Angélica Penteado Dias (Departamento de Ecologia e Biologia Evolutiva da Universidade de São Carlos) pela ajuda na identificação dos parasitóides e à Fundação Rio-Zoo por ter permitido a realização de coletas, em especial ao Biólogo Anderson e ao Sr. Quirino. A CAPES pela bolsa de mestrado concedida ao primeiro autor, a FINEP/CTINFRA-PQ-BIOS (convênio no. 01.03.0068.00) pelo apoio financeiro.

\section{REFERÊNCIAS}

Aguiar-Coelho, V. M.; M. M. C. Queiroz \& E. M. V. Milward-de-Azevedo. 1995. Associação entre larvas de Cochliomyia macellaria (Fabricius) e Chrysomya albiceps (Wiedemann) (Calliphoridae, Diptera) em condições de laboratório. Revista Brasileira de Zoologia 12: 983-990.

Aguiar-Coelho, V.M.\&E.M. V. Milward-de-Azevedo. 1995. Associação entre larvas de Chrysomya megacephala (Fabricius) e Chrysomya albiceps (Wiedemann), Chrysomya megacephala (Fabricius) e Cochliomyia macellaria (Fabricius) (Calliphoridae, Diptera) sob condições de laboratório. Revista Brasileira de Zoologia 12: 991-1000.

Aguiar-Coelho, V. M. \& E. M. V. Milward-de-Azevedo. 1996, Associação entre larvas de Cochliomyia macellaria (Fabricius)e Chrysomya albiceps (Wiedemann), Chrysomya megacephala (Fabricius) e Cochliomyia macellaria (Fabricius) (Calliphoridae, Diptera) sob condições de laboratório. Revista Brasileira de Entomologia 41: 35-40.

Barbosa, L. S.; M. S. Couri \& V. M. Aguiar Coelho. 2008 a. Desenvolvimento de Nasonia vitripennis (Walker, 1836) (Hymenoptera: Pteromalidae) em pupas de Cochliomyia macellaria (Fabricius, 1775) (Diptera: Calliphoridae), utilizando diferentes densidades do parasitóide. Biota Neotropica 8: 49-54.

Barbosa, L. S.; M. S. Couri \& V. M. Aguiar Coelho. 2008 b. Influência do aumento do número de pupas hospedeiras de Cochliomyia macellaria (Diptera, Calliphoridae) no desenvolvimento do parasitóide Nasonia vitripennis (Hymenoptera, Pteromalidae) em laboratório. Iheringia, Sér. Zool. 98: 339-344.

Baumgartner, D. L. 1988. Spread of introduced Chrysomya blowflies (Diptera: Calliphoridae) in the neotropical with records new to Venezuela. Biotropica 20: 167-168.

Baumgartner, D. L. \& B. Greenberg. 1984. The genus Chrysomya (Diptera: Calliphoridae) in the New World. Journal of Medical Entomology 21: 105-113.

Cardoso, D. \& E. M. V. Milward-de-Azevedo. 1995. Influência da densidade de Chrysomya megacephala (Fabricius) (Diptera: Calliphoridae) sobre a capacidade reprodutiva de fêmeas nulíparas de Nasonia vitripennis (Walker) (Hymenoptera: Pteromalidae). Revista Brasileira de Entomologia 39: 779-786.

Cardoso, D. \& E. M. V. Milward-de-Azevedo. 1996. Aspectos da biologia 
de Nasonia vitripennis (Walker) (Hymenoptera: Pteromalidae) em pupas de Chrysomya megacephala (Fabricius) e Chrysomya albiceps (Wiedemann) (Diptera: Calliphoridae), sob condições de laboratório. Revista Brasileira de Entomologia 40: 143-146.

d'Almeida, J. M. \& H. S. Lopes. 1983. Sinantropia de dípteros caliptrados (Calliphoridae) no Estado do Rio de Janeiro. Arquivo da Universidade Federal Rural do Rio de Janeiro. 6: 39-48.

Gagné, R. J. 1981. Chrysomya spp., Old World blow flies (Diptera: Calliphoridae), recently established in the Americas. Bulletin of the Entomological Society of America 27: 21-22.

Guimarães, J. H.; A. P. Prado \& G. M. Buralli. 1979. Dispersal and distribution of three newly introduced species of Chrysomya Robineau-Desvoidy in Brazil (Diptera, Calliphoridae). Revista Brasileira de Entomologia. 23: $245-255$.

Gulias-Gomes, C. C.; C. O. Soares \& E. M. V. Milward-de-Azevedo. 2003. Pupas de Chrysomya megacephala (Fabricius, 1794) (Diptera, Calliphoridae) crioconservadas em soluções de glicerol e dimetil sulfóxido como substrato de criação de Nasonia vitripennis (Walker, 1836) (Hymenoptera, Pteromalidae). Revista Brasileira de Zoociências. 5: $101-120$

Hamilton, W. D. 1967. Extraordinary sex ratios. Science 156: 477-488.

Magalhães, M. N. \& A. C. P. Lima. 2001. Noções de Probabilidade e Estatística. 3 ed. São Paulo IME-USP, 392 p.

Marinho C. R.; A. C. G. Azevedo \& V. M. Aguiar-Coelho, 2003. Diversidade de califorídeos (Diptera: Calliphoridae) em área urbana, Rio de Janeiro.
Entomologia y Vectores, 10: 185-199.

Prado, A. P. 1982. Estudo atual de dispersão e distribuição do gênero Chrysomya Robineau-Desvoidy na região neotropical (Diptera, Calliphoridae). Revista Brasileira de Entomologia 26: 225-231.

Saunders, D. S.; D. Sutton \& R. A. Jarvis. 1970. The effect of host species on diapause induction in Nasonia vitripennis. Journal of Insect Physiology 16: $405-416$.

Shuker, D. M.; I. Pen; A. B. Duncan; S. E. Reece \& A. S. West. 2005. Sex Ratios under Asymetrical Local Mate Competition: Theory and a Test with Parasitoid Wasps. The American Naturalist 166: 301-316.

Van Den Assem, J.; F. A. Putters \& T. C. Prins. 1984. Host quality effects on sex ratio of the parasitic wasp Anisopteromalus calandrae (Chalcidoidae: Pteromalidae). Netherlands Journal of Zoology 34: 33-62.

Walker, I. \& D. Pimentel. 1966. Correlation between maternal longevity and incidence of diapause in Nasonia vitripennis Walker (Hymenoptera: Pteromalidae). Gerontologia 12: 89-98.

Whiting, A. R. 1967. The biology of the parasitic wasp Mormoniella vitripennis (= Nasonia vitripennis) (Walker). Quarterly Review of Biology 42: 333-406.

Wylie, H. G. 1963. Some effects of host age on parasitism by Nasonia vitripennis (Walk.) (Hymenoptera: Pteromalidae). The Canadian Entomologist 95: 881-886.

Wylie, H. G. 1966. Survival and reproduction of Nasonia vitripennis (Walker) on different host population densities. The Canadian Entomologist 98: 273-286. 